\title{
Associations between age and sleep apnea risk among newborn infants
}

\section{Lisa B. Matlen $\mathrm{MD}^{1,2}$ ( $\mid$ Fauziya Hassan MD, MS ${ }^{1,2} \mid$ Renée A. Shellhaas MD, MS}

${ }^{1}$ Department of Pediatrics, University of Michigan, Ann Arbor, Michigan

${ }^{2}$ Michael S. Aldrich Sleep Disorders Center, University of Michigan, Ann Arbor, Michigan

\section{Correspondence}

Lisa Matlen MD, 12-733, CS Mott Children's Hospital, 1540 E Hospital Dr., Ann Arbor, MI 48109-4279.

Email: Imatlen@med.umich.edu

\begin{abstract}
Objective: Among older children, sleep-disordered breathing (SDB) is associated with measurable neurocognitive consequences. However, diagnostic SDB thresholds are lacking for infants $<12$ months. We sought to evaluate the relationship between SDB indices, gestational age (GA), and postmenstrual age (PMA) for infants who underwent clinically-indicated polysomnograms at a tertiary care center.

Methods: Every infant $<3$-months chronological age whose first clinically-indicated polysomnogram was between 2/2012 and 2/2017 was included. Linear regression was used to evaluate associations between apnea-hypopnea index (AHI), obstructiveapnea index (OAI), and GA and PMA for infants with and without obvious clinical risk factors for SDB (eg, micrognathia and cleft palate).

Results: For 53 infants without obvious SDB risk factors (GA $35.6 \pm 4.5$ weeks; PMA $41.2 \pm 4.0$ weeks), mean AHI was $27 \pm 18$ and OAI $2.9 \pm 4.5$. There was a weak inverse relationship between $\mathrm{AHI}$ and $\mathrm{PMA}\left(r^{2}=0.12, P=0.01\right)$, but $\mathrm{AHI}$ was not predicted by GA $\left(r^{2}=0.04, P=0.13\right)$. Conversely, OAl was more strongly associated with GA $\left(r^{2}=0.33, P<0.0001\right)$ than PMA $\left(r^{2}=0.08, P=0.036\right)$. For 28 infants with congenital structural anomalies that predispose to SDB (GA 38.0 \pm 3.1 weeks, PMA $43.1 \pm 3.3$ weeks, AHI $37.7 \pm 30$, OAI $8.2 \pm 11.8$ ), neither AHI nor OAI were related to PMA or GA.

Conclusions: Among infants who received clinically-indicated polysomnograms but did not have obvious structural risk for SDB, AHI declined with advancing PMA, but obstructive-apnea was best predicted by prematurity. In contrast, the SDB risk did not improve with increasing GA or PMA for infants with congenital structural risk factors; such infants may not outgrow their risk for SDB.
\end{abstract}

\section{KEYWORDS}

central sleep apnea, hypoventilation, obstructive sleep apnea, polysomnography,

sleep-disordered breathing
This project was not supported by external funding sources. The authors declare that they have no conflicts of interest.

This study was presented in abstract form at the annual meeting of the Child Neurology Society (Chicago, IL, October 2018).

\section{1 | INTRODUCTION}

Sleep is a powerful indicator of global neurological function; a recent study of neonates with risk for cerebral dysfunction reported that objective sleep measures may independently predict 18 to 22 -month neurodevelopmental outcomes. ${ }^{1}$ Healthy full-term neonates have 
higher frequency of apneas and hypopneas than older children (mean neonatal apnea-hypopnea index, $\mathrm{AHI}$, was reported to be $14.9 \pm 9.1$ among 30 healthy infants, ${ }^{2}$ with neonatal obstructive AHI $2.3 \pm 2.5$, while obstructive $\mathrm{AHI}>1$ is considered abnormal for children $>12$ months of age). ${ }^{3,4}$ Despite clinical observations of sleep-disordered breathing (SDB) patterns on polysomnography, parameters to guide clinically appropriate diagnostic limits for SDB are not currently welldefined for neonates or infants $<1$ year. $^{5}$

Infants who are born prematurely are predisposed to adverse cognitive outcomes ${ }^{6}$ and appear to be at risk for later SDB. ${ }^{7}$ Habitual snoring with associated arousals, even in the absence of measurable sleep apnea, among infants is associated with suboptimal motor developmental scores. ${ }^{8}$ This suggests that the impact of even mild or subthreshold severity symptoms of SDB may be clinically significant. As symptoms of SDB are associated with later neurobehavioral consequences, ${ }^{9}$ and are potentially treatable, establishing threshold definitions for SDB in infants should be a priority for clinicians and researchers. To begin to fill this important knowledge gap, we sought to evaluate the relationship between SDB indices and gestational age (GA) at birth and postmenstrual age (PMA) at the time of the polysomnogram for infants at a tertiary care center.

We hypothesized that premature infants without structural airway abnormalities would have higher $\mathrm{AHI}$ than term infants, ${ }^{7}$ but that their risk for SDB would decline with increasing PMA. The risk for SDB may decline as the infant grows due to reduction in chest wall compliance and developmental maturity of respiratory control. Central sleep apnea indices have been observed to decline over time in healthy term infants. ${ }^{10}$ Thus, we also expected an inverse relationship between central apnea index (CAI) and PMA in this cohort.

Secondarily, we predicted that infants with congenital structural airway anomalies would have higher obstructive-apnea indices (OAI) than those without obvious risk factors, while infants with suspected or confirmed genetic syndromes (which often impact central respiratory drive and may be associated with hypotonia) would have higher CAI and risk for hypoventilation than nonsyndromic infants. We anticipated that all neonates would have declining SDB indices with increasing PMA.

\section{2 | METHODS}

This study was approved by our Institutional Review Board and a waiver of informed consent was granted.

Subjects: all infants of chronological age $<3$ months who underwent their first clinically-indicated, attended polysomnogram between 2/2012 and 2/2017 at Michigan Medicine were included. Data were abstracted via systematic medical record review and included GA, PMA at time of the first polysomnogram, sex, birthweight, Apgar scores, medical comorbidities, indication for polysomnography, standard polysomnographic results, SDB-related diagnoses, and treatment or interventions recommended after the polysomnogram. Infants who were predisposed to SDB due to suspected or confirmed genetic or craniofacial abnormalities, structural airway abnormalities, known structural brain abnormalities, such ventriculomegaly, or a diagnosed congenital nervous system malformation, were analyzed as a subgroup.

Exclusion criteria were as follows: patients who had undergone polysomnography solely for research (not due to clinical suspicion for SDB), incomplete information about the patient's gestational age at birth in the medical record, patients whose first polysomnogram was performed at a different institutions.

Polysomnography: attended polysomnography at our institution includes six channel electroencephalography (F3/F4, C3/C4, and O1/ O2 electrode positions), two electrocardiogram channels, electrooculogram, chin surface electromyography (EMG), bilateral anterior tibialis EMG, chest and abdominal excursion, nasal pressure, nasal/ oral airflow (with thermal detection), a snoring sensor, oxygen saturation by pulse oximetry, and transcutaneous carbon dioxide monitoring. Video monitoring was recorded simultaneously. Clinical polysomnography software was used (Compumedics, Victoria, Australia). Sleep-wake stage scoring was performed based on a combination of behavioral observation and recorded polysomnography data, according to contemporaneous standard neonatal scoring rules. ${ }^{11,12}$ Studies before July 1, 2015 scored sleep-wake stages by 30 -second epochs as wakefulness, quiet, active, or indeterminate sleep. ${ }^{11}$ Subsequent studies were scored using the 2015 scoring criteria by 30 -second epochs of wakefulness, nonrapid eye movements (NREM; analogous to quiet sleep), REM (analogous to active sleep), and transitional sleep (analogous to indeterminate sleep). ${ }^{12}$

Respiratory event scoring rules followed published pediatric criteria. ${ }^{13}$ Obstructive-apnea was defined as $>90 \%$ decrement in airflow for the duration of at least two breaths with preserved respiratory effort. Central apnea was identified by absence of inspiratory effort and one of the following: duration $>20$ seconds; duration of two or more breaths and associated arousal or $>3 \%$ oxygen desaturation; duration of at least two breaths and associated heart rate $<50$ beats per minute for at least 5 seconds or $<60$ beats per minute for at least 15 seconds. Hypopneas were scored based on peak signal excursion decrement of $>30 \%$, lasting at least two breaths, and associated with at least one of $a \geq 3 \%$ oxygen desaturation or an arousal. Hypopneas were not further classified into central hypopneas or obstructive hypopneas. The apneahypopnea index (AHI) was calculated as the total number apneas and hypopneas per hour of sleep. The obstructive-apnea index (OAI) included only obstructive apneas, while the central apnea index (CAI) included only central apneas per hour of sleep; neither of these subindices included hypopneas. Time spent with oxygen saturation $<90 \%$ was calculated from valid pulse oximetry reading during sleep, excluding movement artifact. Hypoventilation was scored

when $>25 \%$ of the total sleep time was spent with carbon dioxide monitoring $>50 \mathrm{~mm} \mathrm{Hg}$ as measured by transcutaneous carbon dioxide monitoring $\left(\mathrm{TCO}_{2}\right)$. Given the lack of established thresholds for infants, a diagnosis of obstructive sleep apnea was provided at the discretion of the interpreting provider; the diagnosis was 
typically assigned when the OAl was higher than 1. Similarly, a diagnosis of central sleep apnea was provided at the discretion of the interpreting provider when the central apnea index was more than 5 . Primary central sleep apnea of infancy is defined by the ICSD-3 (International Classification of Sleep Disorders, third edition) as apnea or cyanosis noted by an observer or sleep-related central apnea or oxygen desaturation detected by monitoring in an infant at least 37 weeks' gestation, with PSG demonstrating either recurrent episodes of central apneas $>20$ seconds or periodic breathing for $5 \%$ of the sleep time, not better explained by another disorder or by medication. ${ }^{14}$ All polysomnograms were scored by an experienced, registered polysomnographic technologist, and reviewed by a boardcertified pediatric sleep medicine physician.

Analyses: multivariate regression was used to analyze associations of GA and PMA with polysomnogram data including $\mathrm{AHI}, \mathrm{OAI}, \mathrm{CAI}$, percentage of time with baseline oxygen saturation $\left(\% \mathrm{O}_{2}\right)<90 \%$, and diagnosis of hypoventilation. Fisher exact test was performed to compare categorical groups of infants with specific SDB diagnoses. One-way analysis of variance (ANOVA) tests and Kruskal-Wallis tests for normal and skewed distributions, respectively, were used to compare the polysomnographic data among groups of infants with SDB diagnoses. Analyses were performed using SAS (Cary, NC).

\section{3 | RESULTS}

Among the 124 unique infants included in the initial chart review, five were excluded due to lack of documented GA at birth and one was excluded due to an outlier $\mathrm{AHI}$ value (136). Among the remaining 118 infants (Table 1), indications for polysomnography included: apneic episodes (53), oxygen desaturation (43), suspected, or diagnosed upper airway anomaly (eg, laryngomalacia and Pierre Robin sequence) (31), apparent life-threatening event or brief resolved unexplained event (ALTE/BRUE) (16), cyanotic events (10), noisy breathing or snoring (10), hypotonia (7), respiratory failure/ tracheostomy (7), and clinical hypoventilation (4) (Figure 1). Note that some infants had more than one indication for their first polysomnogram.

Diagnoses derived from the polysomnograms included obstructive sleep apnea (OSA) (67), central sleep apnea (CSA) (47), primary central apnea of infancy (24), and hypoventilation (21). Recommended treatments included clinic follow-up (77), repeat PSG (70), oxygen therapy (67), medication (58: caffeine, ranitidine, or proton pump inhibitor), and surgery (35: eg, distraction osteogenesis, tracheostomy, supraglottoplasty, and vallecular cyst removal).

Among the 118 infants (Table 1), 53 had no obvious risk factors for SDB (GA 35.6 \pm 4.5 weeks; PMA $41.2 \pm 4.0$ weeks) and their mean $\mathrm{AHI}$ was $27 \pm 18$ and $\mathrm{OAI} 2.9 \pm 4.5$. There was a weak inverse relationship between $\mathrm{AHI}$ and $\mathrm{PMA}$ for this group of infants $\left(r^{2}=0.12, P=0.01\right)$ (Figure 2D), but AHI was not predicted by GA $\left(r^{2}=0.04, P=0.13\right)$ (Figure 2C). Conversely, OAI was much more strongly associated with $\mathrm{GA}\left(r^{2}=0.33, P<0.0001\right)$ (Figure $\left.2 \mathrm{~A}\right)$ than PMA $\left(r^{2}=0.08, P=0.036\right)$ (Figure $2 B$ ). Clinical variables including birthweight $(P=0.79)$, sex $(P=0.53)$, and 5-minute Apgar score $(P=0.11)$ were not associated with $\mathrm{AHI}$.

There were 28 infants with structural airway anomalies: isolated cleft lip/palate (8), Pierre Robin Sequence (7), other structural abnormalities (7: eg, laryngomalacia and micrognathia), and combination of an airway abnormality and a genetic abnormality (6). Henceforth, this group of 28 infants will be labeled the "airway" group. In addition, there were 31 infants with a confirmed or suspected genetic syndrome, termed the "genetic" group. Genetic diagnoses included pathological mutation in $\mathrm{PHOX} 2 \mathrm{~B}$ gene (1), congenital myotonic dystrophy (1), DiGeorge syndrome (4), trisomy 21 (2), trisomy 18 (3), spinal muscular atrophy (2), Prader Willi (2), VACTERL syndrome (vertebral defects, anal atresia, cardiac defects, trachea-esophageal fistula, renal anomalies, and limb abnormalities) (1), Beckwith-Wiedemann (1), Rubinstein-Taybi (1), Cri-du-chat (1), pathological MECP2 variant (1), Kabuki syndrome (1), myopathy (1), and various chromosomal deletion syndromes (1p36, 7q21.11, 14q, 15q11, and 16p13.11), duplications (1q44, 8, 20), along with suspected syndromes by facial dysmorphism or other multiorgan anomalies suspicious for genetic syndrome. Six additional infants were included which were assessed to have predisposing risk factors for SDB yet did not fit cleanly into the other groups: myelomeningocele, grade III-IV intraventricular hemorrhage, hydrocephalus statuspost implantation of ventriculo-peritoneal shunt, and bronchopulmonary dysplasia. Among the 28 patients in the "airway" group (GA $38.0 \pm 3.1$ weeks, PMA 43.1 \pm 3.3 weeks, AHI $37.7 \pm 30$, OAI $8.2 \pm 11.8$ ), neither $\mathrm{AHI}$ nor OAI were related to PMA or GA.

Neither GA nor PMA was associated with oxygen desaturation (percent of time with oxygen saturation $<90 \%$ ) in the full sample, nor in the "no obvious risk factors," "airway," or "genetic" subgroups (Table 2). However, the percent of time with oxygen saturation $<90 \%$ varied significantly among the subgroups (ANOVA $P=0.006$ ), with less time with oxygen saturation $<90 \%$ among infants without clear SDB risk factors compared with the "airway" $(P=0.001)$ and "genetic" $(P=0.001)$ subgroups (Table 1 ).

Likewise, neither CAI nor hypoventilation were associated with GA or PMA in any of the groups (Table 2). Compared with the infants without SDB risk factors, the proportion of infants with hypoventilation was higher among the "airway" group (Fisher exact test $P=0.006$ ) and those with genetic diagnoses $(P<0.0001)$. CAl varied among patient groups (ANOVA $P=0.01$ ). Infants without obvious SDB risk factors had higher CAI than those with genetic diagnoses. OAI did not vary among the three groups of infants (ANOVA $P=0.51$ ).

\section{4 | DISCUSSION}

These study results suggest that the natural history of SDB may change during the first months of life. Among infants without obvious predisposing risk factors for SDB, advancing PMA (eg, evolving maturity after birth) was associated with lower AHI. However, prematurity alone (low GA) did not predict $\mathrm{AHI}$ in the first 3 months after birth. 
TAB LE 1 Clinical and demographic data for 118 infants aged $<3$ months who had a clinically-indicated polysomnogram. Data are provided as mean \pm standard deviation unless otherwise indicated

\begin{tabular}{|c|c|c|c|c|c|c|}
\hline Characteristics & Total & $\begin{array}{l}\text { No obvious risk } \\
\text { for } \text { SDB }^{d}\end{array}$ & $\begin{array}{l}\text { Airway } \\
\text { anomalies }^{a}\end{array}$ & $\begin{array}{l}\text { Genetic } \\
\text { diagnosis }^{b}\end{array}$ & Other ${ }^{c}$ & p-value \\
\hline$N$ & 118 & 53 & 28 & 31 & 6 & \\
\hline $\mathrm{PMA}^{\mathrm{d}}$ at time of $\mathrm{PSG}^{\mathrm{d}}$ (wk) & $42.5 \pm 3.9$ & $41.2 \pm 4.0$ & $43.1 \pm 3.3$ & $44.2 \pm 3.6$ & $42.7 \pm 4.8$ & $P=0.0065$ \\
\hline $\begin{array}{l}\text { Chronological age at time of PSG } \\
\text { (d) }\end{array}$ & $40.4 \pm 26.8$ & $39.5 \pm 26.5$ & $35.7 \pm 24.4$ & $46.6 \pm 27.1$ & $38.3 \pm 39.2$ & $P=0.46$ \\
\hline Gender (F) \# (\%) & $61(52 \%)$ & 27 (51\%) & $13(46 \%)$ & $18(58 \%)$ & $3(50 \%)$ & $P=0.85$ \\
\hline $\begin{array}{l}\text { 5-min Apgar score - median } \\
\text { [interquartile range] }\end{array}$ & $9[1.25]$ & 9 [1] & 8 [1.25] & $8[2]$ & $7.5[1.75]$ & $P=0.37$ \\
\hline Total sleep time (TST) (min) & $374.27 \pm 83.9$ & $378.8 \pm 81.2$ & $351.6 \pm 63.9$ & $349.9 \pm 76.3$ & $439.7 \pm 148.7$ & $P=0.04$ \\
\hline \multicolumn{7}{|l|}{ AHI N (\%) per group: } \\
\hline $3-5$ & $4(3.4 \%)$ & $2(3.8 \%)$ & $0(0 \%)$ & $2(6.5 \%)$ & $0(0 \%)$ & \\
\hline $5-10$ & $16(13.6 \%)$ & $6(11.3 \%)$ & $5(17.9 \%)$ & $5(16.1 \%)$ & $0(0 \%)$ & \\
\hline $10-20$ & $38(32.2 \%)$ & $17(32.1 \%)$ & $5(17.9 \%)$ & $14(45.2 \%)$ & $2(33.3 \%)$ & \\
\hline $20-30$ & 13 (11.0\%) & $8(15.1 \%)$ & $3(10.7 \%)$ & $1(3.2 \%)$ & $1(16.7 \%)$ & \\
\hline $30-40$ & 11 (9.32\%) & 7 (13.2\%) & $2(7.1 \%)$ & $2(6.5 \%)$ & $0(0 \%)$ & \\
\hline$>40$ & $36(30.5 \%)$ & $13(24.5 \%)$ & $13(46.4 \%)$ & 7 (22.6\%) & $3(50.0 \%)$ & \\
\hline$O A I^{d}$ & $4.7 \pm 8.4$ & $2.9 \pm 4.5$ & $8.2 \pm 11.8$ & $4.8 \pm 9.6$ & $3.1 \pm 4.9$ & $P=0.51$ \\
\hline $\mathrm{CAl}^{\mathrm{d}}$ & $7.2 \pm 10.96$ & $8.4 \pm 11.6$ & $5.3 \pm 7.8$ & $6.4 \pm 12.9$ & $10.0 \pm 7.0$ & $P=0.01$ \\
\hline
\end{tabular}

Note: $\mathrm{AHI}$ is calculated as total apneas + hypopneas divided by total sleep time. OAl is calculated by obstructive apneas divided by total sleep time. CAI is calculated by central apneas divided by total sleep time.

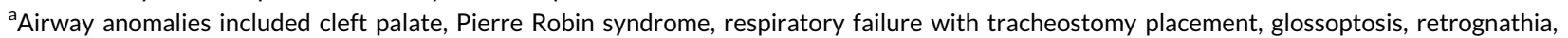
micrognathia, and laryngomalacia.

${ }^{\mathrm{b}}$ Genetic syndromes included central congenital hypoventilation syndrome, spinal muscular atrophy, congenital myotonic dystrophy, trisomy 18 , trisomy 21, Prader Willi, Beckwith-Widemann, Rubinstein-Taybi, and pathogenic variant in MECP2 gene.

${ }^{\mathrm{c}}$ Included those at risk for SDB, for example, myelomeningocele, grade III-IV intraventricular hemorrhage and hydrocephalus s/p VP shunt, bronchopulmonary dysplasia.

${ }^{\mathrm{d}} \mathrm{AHI}$, apnea-hypopnea index; CAI, central apnea index; OAI, obstructive-apnea index; PMA, postmenstrual age; PSG, polysomnogram; SDB, sleepdisordered breathing.

$\mathrm{AHI}$ is calculated by combining both obstructive and central apneas into one calculation, but risks for each appear to be separate. We demonstrate a strong inverse association between OAI and GA, while the relationship between OAI and PMA is much less robust. These findings suggest that infants born prematurely (low GA) have static risk factors for obstructive respiratory events which do not correct with evolving maturity. However, risk for SDB did not change with prematurity or postnatal age among infants with predisposing structural or genetic factors. For these subgroups of infants, the protective factors of advancing age (PMA) and developmental status may be masked by the SDB risk conferred by their congenital risk abnormalities. Thus, their SDB risk may not be simply outgrown over time. This set of novel findings has direct implications for SDB treatment: interventions may be appropriate sooner for children with structural or genetic SDB risk factors than for those without obvious anomalies as the former group is unlikely to outgrow their SDB with watchful waiting.

Early treatment for SDB may be particularly important for neurodevelopmental outcomes. Snoring was not directly assessed in our study but is a risk factor for SDB. Parent-reported snoring in the first 4 weeks of life predicts snoring at 6 months of age. ${ }^{15}$ Even the 


\section{Clinical Indications for PSG}

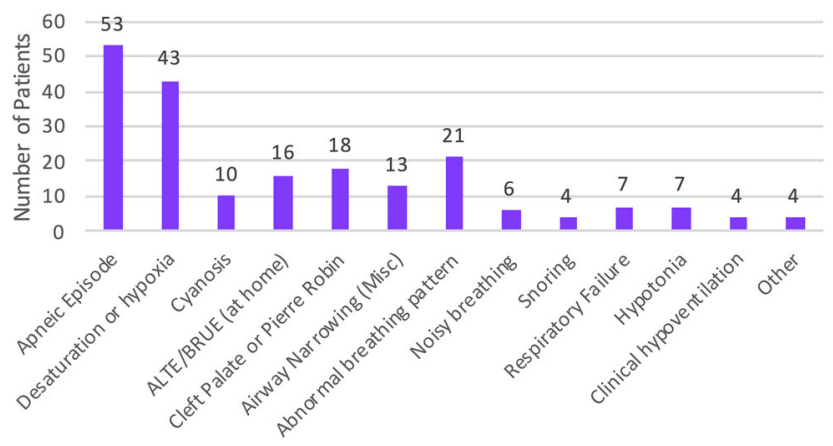

FIGURE 1 Indications for neonatal polysomnography for 118 consecutive infants $<3$ months of age [Color figure can be viewed at wileyonlinelibrary.com]

presence of snoring-related arousals without demonstrable elevations in $\mathrm{AHI}(\mathrm{AHI}<1)$ has been associated with reduced Bayley scores in infants. ${ }^{8}$ Snoring is commonly understood to fall along the same disease spectrum as obstructive sleep apnea. ${ }^{16}$ Although
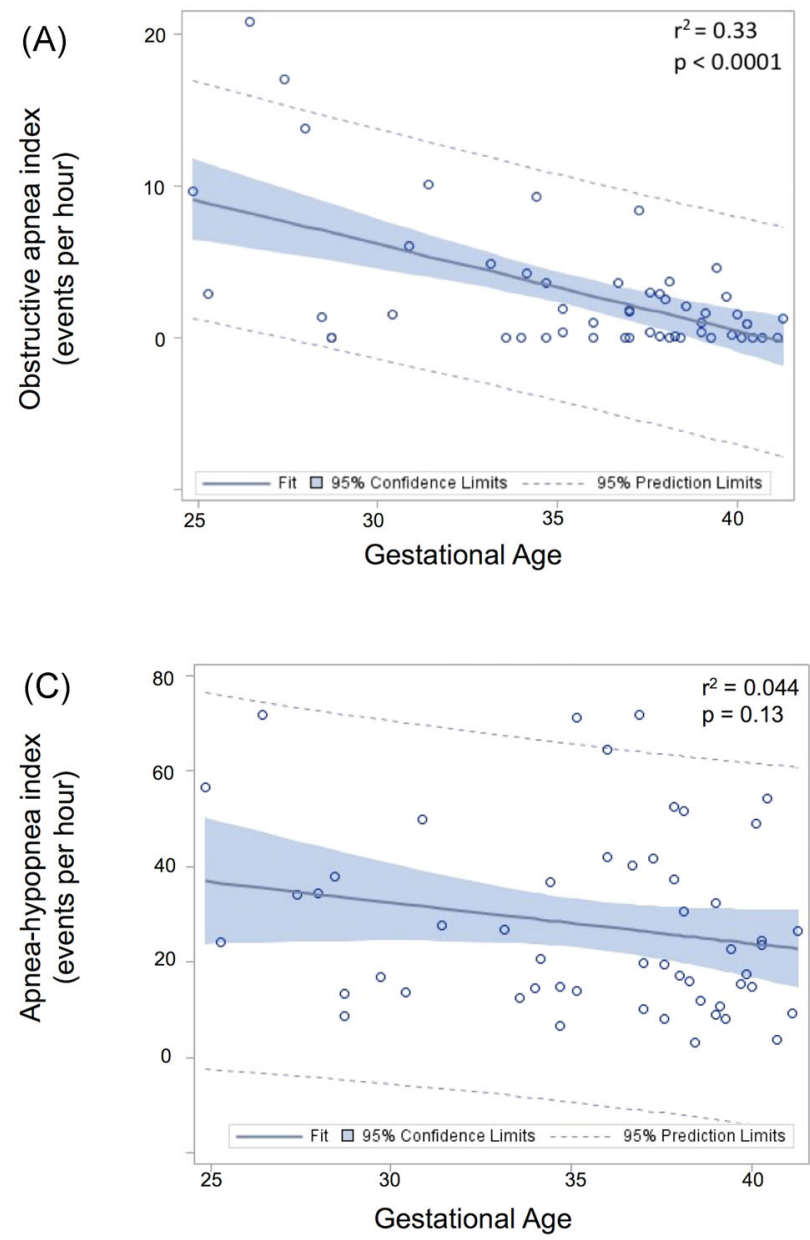

abnormal $\mathrm{AHI}$ thresholds have not yet been determined in infants, it is hypothesized based on adult data that a higher AHI may correlate with a higher disease burden. How a highly elevated $\mathrm{AHI}$ (or OAI, CAI), as was observed in our study, even in infants without obvious risk factors for SDB, may correlate with risk for abnormal neurodevelopmental outcomes is currently uncertain. This is an important target for future research.

In a study of 30 healthy, term infants who had PSGs at a chronological age of less than 30 days, mean $\mathrm{AHI}$ was $14.9 \pm 9.1$ and both CAI $(5.4 \pm 6.2)$ and OAI $(2.3 \pm 2.5)$ were much higher than established normal thresholds for children older than 12 months. ${ }^{2}$ While results from a limited sample of healthy neonates cannot be translated directly into diagnostic threshold recommendations, especially for infants with medical complexity, the data provide additional evidence that standards for older children should not be extrapolated to neonates. Comparing these results for normal infants with the findings in our cohort suggests that neonates with medical comorbidities may often have significantly elevated $\mathrm{AHI}, \mathrm{CAl}$, and OAI.

We report no associations between prematurity (low GA) or postnatal development (PMA) and CAI, diagnosis of hypoventilation,
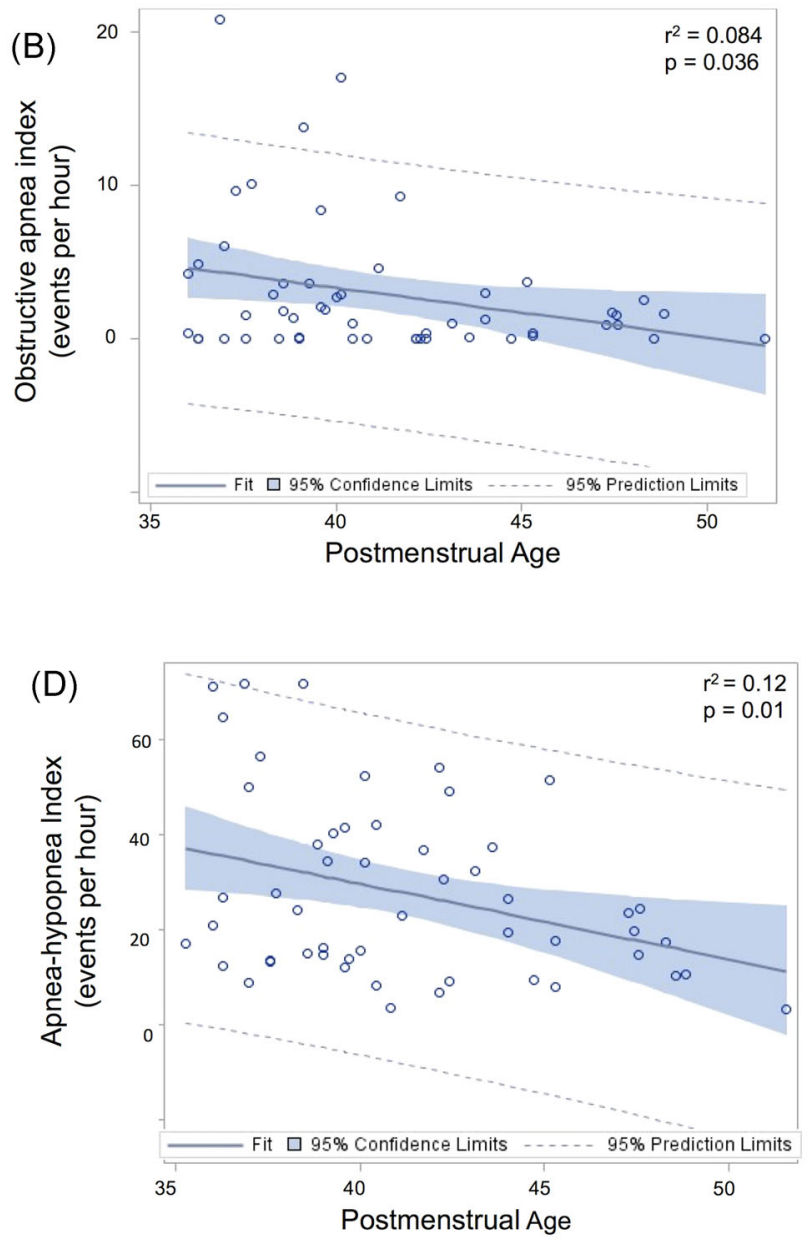

FIGURE 2 Among 53 infants without obvious risk factors for sleep-disordered breathing, obstructive-apnea index declined more robustly with gestational age at birth $\left(\mathrm{A}, r^{2}=0.33, P<0.0001\right)$ than postmenstrual age $\left(\mathrm{B}, r^{2}=0.08, P=0.036\right)$. Conversely, apnea-hypopnea index did not vary with gestational age $\left(C, r^{2}=0.04, P=0.13\right)$, but declined modestly with increasing postmenstrual age $\left(D, r^{2}=0.12, P=0.01\right)[C o l o r$ figure can be viewed at wileyonlinelibrary.com] 
TABLE 2 Bivariate analysis models of SDB risk for 118 infants $<3$ months of age who had a clinically-indicated polysomnogram

\begin{tabular}{|c|c|c|c|}
\hline Characteristics & Total & $\begin{array}{l}\text { No obvious } \\
\text { risk }\end{array}$ & $\begin{array}{l}\text { Airway } \\
\text { anomalies }\end{array}$ \\
\hline $\mathrm{N}$ & 118 & 53 & 28 \\
\hline \multicolumn{4}{|l|}{ AHI: } \\
\hline GA & $\begin{aligned} R^{2} & =0.0021 \\
P & =0.62\end{aligned}$ & $\begin{aligned} R^{2} & =0.044 \\
P & =0.13\end{aligned}$ & $\begin{aligned} R^{2} & =0.12 \\
P & =0.07\end{aligned}$ \\
\hline PMA & $\begin{aligned} R^{2} & =0.070 \\
P & =0.0037\end{aligned}$ & $\begin{array}{r}R^{2}=0.12 \\
P=0.01\end{array}$ & $\begin{aligned} R^{2} & =0.085 \\
P & =0.13\end{aligned}$ \\
\hline \multicolumn{4}{|l|}{ OAI } \\
\hline GA & $\begin{aligned} R^{2} & =0.045 \\
P & =0.021\end{aligned}$ & $\begin{aligned} R^{2} & =0.33 \\
P & <0.0001\end{aligned}$ & $\begin{aligned} R^{2} & =0.076 \\
P & =0.16\end{aligned}$ \\
\hline PMA & $\begin{aligned} R^{2} & =0.012 \\
P & =0.23\end{aligned}$ & $\begin{aligned} R^{2} & =0.084 \\
P & =0.036\end{aligned}$ & $\begin{aligned} R^{2} & =0.007 \\
P & =0.66\end{aligned}$ \\
\hline \multicolumn{4}{|l|}{ CAI } \\
\hline GA & $\begin{aligned} R^{2} & =0.004 \\
P & =0.48\end{aligned}$ & $\begin{aligned} R^{2} & =0.014 \\
P & =0.40\end{aligned}$ & $\begin{aligned} R^{2} & =0.029 \\
P & =0.39\end{aligned}$ \\
\hline PMA & $\begin{aligned} R^{2} & =0.012 \\
P & =0.23\end{aligned}$ & $\begin{aligned} R^{2} & =0.0005 \\
P & =0.89\end{aligned}$ & $\begin{aligned} R^{2} & =0.006 \\
P & =0.70\end{aligned}$ \\
\hline \multicolumn{4}{|l|}{$\% \mathrm{O}_{2}<90 \%$} \\
\hline GA & $\begin{aligned} R^{2} & =0.0008 \\
P & =0.80\end{aligned}$ & $\begin{array}{r}R^{2}=0.05 \\
P=0.11\end{array}$ & $\begin{aligned} R^{2} & =0.008 \\
P & =0.65\end{aligned}$ \\
\hline PMA & $\begin{aligned} R^{2} & =0.005 \\
P & =0.45\end{aligned}$ & $\begin{aligned} R^{2} & =0.067 \\
P & =0.062\end{aligned}$ & $\begin{aligned} R^{2} & =0.036 \\
P & =0.33\end{aligned}$ \\
\hline \multicolumn{4}{|l|}{ Hypoventilation } \\
\hline GA & $P=0.80$ & Not valid* & $P=0.37$ \\
\hline PMA & $P=0.21$ & Not valid & $P=0.72$ \\
\hline
\end{tabular}

Abbreviations: $\mathrm{AHI}$, apnea-hypopnea index; CAI, central apnea index; GA, gestational age; PMA, postmenstrual age; OAI, obstructive-apnea index; SDB, sleep-disordered breathing.

Note: $\mathrm{AHI}$ is calculated as total apneas + hypopneas divided by total sleep time. OAl is calculated by obstructive apneas divided by total sleep time. CAl is calculated by central apneas divided by total sleep time.

"Not valid due to lack of sufficient sample in this group with diagnosis of hypoventilation such that a linear regression could not be formed.

or oxygen desaturation ( $\% \mathrm{O}_{2}<90 \%$ ) within the first 3 months after birth. This is in contrast to previous work that reported correlation between rising PMA and lower CAI over the first 3 months of life among healthy infants. ${ }^{10,17}$ By contrast, our study population was not composed of healthy controls, but instead comprised of patients for whom there was a clinical indication for polysomnography. In addition, we did not have sufficient sample size of patients with repeat polysomnograms to enable repeated evaluations over time. Our patients may not have reached the PMA at which CAI might be expected to decrease. Despite this, it is notable that oxygen desaturation remained common even among the older infants in our cohort; this may be a reflection of the clinical conditions that triggered the request for polysomnography. In addition, the extent of oxygen desaturation improves with age, ${ }^{18}$ yet the timing of the polysomnography in our cohort may have been too early to demonstrate an improvement with advancing age.
We confirm that infants with airway anomalies may be at particularly high risk for OSA and oxygen desaturation. While CAI was not more prevalent among infants with genetic syndromes than the other groups, the risk for hypoventilation was especially elevated for this subgroup. Though genetic syndromes are heterogeneous, affected infants often have neonatal hypotonia, which may predispose to clinical hypoventilation. Our data suggest that for such infants, clinicians may need to focus particular attention on diagnosis and management of hypoventilation.

PSG-defined diagnoses of hypoventilation ( $>25 \%$ of the total sleep time with $\mathrm{CO}_{2}$ values $>50 \mathrm{~mm} \mathrm{Hg}$ ) is well accepted. Yet, for infants $<12$ months of age, the thresholds for diagnosis of OSA, CSA, and primary central apnea of infancy are not well-defined. There is a clear need to study the associations between neonatal OAI, CAI, and outcomes to determine clinically-meaningful diagnostic criteria and to define thresholds for intervention.

Despite the lack of widespread consensus regarding the clinical diagnosis of SDB in neonates, the results of the clinical polysomnography data reported here, combined with individual infants' clinical histories, triggered clinical interventions for all but nine infants (109/ $118,92 \%$ ). The most common treatment was supplemental oxygen (57\%), but medication (caffeine, ranitidine, or proton pump inhibitor) was prescribed for $49 \%$ and surgery was indicated for $30 \%$ (nearly all of whom had congenital airway anomalies).

In contrast to older children, some respiratory events are expected even among seemingly healthy infants. In one study of 37 healthy full-term infants, the median $\mathrm{AHI}$ fell from 7.8 at 1 month to 4.9 at 3 months and $\mathrm{CAl}$ and percent time with oxygen saturation < $90 \%$ also declined. ${ }^{19}$ A limitation of that study is that unattended home sleep apnea testing without EEG capability to detect sleep staging or arousals was utilized, rather than gold-standard attended polysomnography. In a study of 1023 healthy term infants, median OAl between 2 to 7 weeks was 0.3 , with a declining frequency of obstructive and mixed apneas as the infant was analyzed over time, up to 27 weeks' chronological age. ${ }^{20}$ These studies excluded premature infants, and those with structural airway anomalies or genetic syndromes. In contrast, our cohort instead represented a tertiary care center referral base for clinical suspicion of SDB, which included the infants which were excluded above. This is a likely explanation for the apparently higher rate of polysomnographic abnormalities among our study subjects.

Although we included every infant $\leq 3$ months chronological age who received a clinical, attended, gold-standard polysomnogram during a 5-year timespan, our study has some limitations. The study was retrospective and is subject to the inherent flaws of this design. We are not able to comment on the natural history of polysomnographic results for healthy newborn infants, since all of the included patients had a clinical indication for a sleep evaluation. We did not have a large enough sample of serial polysomnograms to permit trajectory analyses of sleep data over time for individual infants and the potential effect of night-to-night variability is unknown in this age group. It is possible that infants with more severe disease presented chronologically earlier than those with milder SDB. 


\section{5 | CONCLUSIONS}

Despite the lack of established consensus regarding diagnostic thresholds for SDB among infants, we demonstrated that polysomnography commonly triggers treatment for SDB in this age group. Premature infants and those with structural airway anomalies and genetic syndromes appear to have static risk factors for SDB for which early intervention should be targeted; those with genetic syndromes should also be closely monitored for hypoventilation. These results highlight an urgent need for outcomes-based research on SDB in young infants to define diagnostic thresholds and enable studies of therapeutic interventions.

\section{ACKNOWLEDGMENTS}

The authors would like to thank Stephanie Rau, CCRP, and Shannon Lester, CCRP, for their assistance with this project.

\section{ORCID}

Lisa B. Matlen (D) http://orcid.org/0000-0003-3016-1676

\section{REFERENCES}

1. Shellhaas RA, Burns JW, Hassan F, Carlson MD, Barks JD, Chervin RD. Neonatal sleep-wake analyses predict 18-month neurodevelopmental outcomes. Sleep. 2017;40(11). https://doi.org/-org.proxy.lib. umich.edu/10.1093/sleep/zsx144

2. Daftary AS, Jalou HE, Shively L, Slaven JE, Davis SD. Polysomnography reference values in healthy newborns. J Clin Sleep Med. 2019;15(3):437-443.

3. Katz ES, Mitchell RB, D'Ambrosio CM. Obstructive sleep apnea in infants. Am J Respir Crit Care Med. 2012;185(8):805-816.

4. Scholle S, Wiater A, Scholle HC. Normative values of polysomnographic parameters in childhood and adolescence: cardiorespiratory parameters. Sleep Med. 2011;12(10):988-996.

5. DeHaan KL, Seton C, Fitzgerald DA, Waters KA, MacLean JE. Polysomnography for the diagnosis of sleep disordered breathing in children under 2 years of age. Pediatr Pulmonol. 2015;50(12): 1346-1353.

6. Chan E, Leong P, Malouf R, Quigley MA. Long-term cognitive and school outcomes of late-preterm and early-term births: a systematic review. Child Care Health Dev. 2016;42(3):297-312.
7. Tapia IE, Shults J, Doyle LW, et al. Perinatal risk factors associated with the obstructive sleep apnea syndrome in school-aged children born preterm. Sleep. 2016;39:737-742.

8. Montgomery-Downs HE, Gozal D. Snore-associated sleep fragmentation in infancy: mental development effects and contribution of secondhand cigarette smoke exposure. Pediatrics. 2006;117(3):e496-e502.

9. Piteo AM, Kennedy JD, Roberts RM, et al. Snoring and cognitive development in infancy. Sleep Med. 2011;12(10):981-987.

10. Ng DK, Chan CH. A review of normal values of infant sleep polysomnography. Pediatr Neonatol. 2013;54(2):82-87.

11. Anders TF, Emde RN, Parmelee AH, University of California Los Angeles. Brain information service. A manual of standardized terminology, techniques and criteria for scoring of states of sleep and wakefullness in newborn infants. In: Anders Thomas, Emde Robert, Parmelee Arthur, eds. , Los Angeles: UCLA Brain Information Service; 1971. ii, 10, 15 p. p

12. Grigg-Damberger MM. The visual scoring of sleep in infants 0 to 2 months of age. J Clin Sleep Med. 2016;12(3):429-445.

13. Berry RB, Budhiraja R, Gottlieb DJ, et al. Rules for scoring respiratory events in sleep: update of the 2007 AASM manual for the scoring of sleep and associated events. J Clin Sleep Med, 82012:597-619.

14. American Academy of Sleep Medicine. International classification of sleep disorders. 3rd ed. Darien, IL; 2014:94-98.

15. Mitchell EA, Thompson JM. Snoring in the first year of life. Acta Paediatr. 2003;92(4):425-429.

16. Montgomery-Downs HE, Gozal D. Sleep habits and risk factors for sleep-disordered breathing in infants and young toddlers in Louisville, Kentucky. Sleep Med. 2006;7(3):211-219.

17. Schlüter B, Buschatz D, Trowitzsch E Polysomnographic reference curves for the first and second year of life. Somnologie. Volume 5: Steinkopff-Verlag; 2001. p 3-16.

18. Terrill PI, Dakin C, Hughes I, Yuill M, Parsley C. Nocturnal oxygen saturation profiles of healthy term infants. Arch Dis Child. 2015; 100(1):18-23.

19. Brockman PE, Poets A, Poets CF. Reference values for respiratory events in overnight polygraphy from infants aged 1 and 3 months. Sleep Med. 14. Elsevier B.V; 2013:1323-1327.

20. Kato I, Franco P, Groswasser J, Kelmanson I, Togari H, Kahn A. Frequency of obstructive and mixed sleep apneas in 1,023 infants. Sleep. 2000;23(4):487-492.

How to cite this article: Matlen LB, Hassan F, Shellhaas RA. Associations between age and sleep apnea risk among newborn infants. Pediatric Pulmonology. 2019;54:1297-1303. https://doi.org/10.1002/ppul.24354 